\title{
BMJ Open Revealed versus concealed criteria for placental insufficiency in an unselected obstetric population in late pregnancy (RATIO37): randomised controlled trial study protocol
}

Francesc Figueras, ${ }^{1,2}$ Eduard Gratacos, ${ }^{1,2}$ Marta Rial, ${ }^{1,2}$ Ilan Gull, ${ }^{3}$ Ladislav Krofta, ${ }^{4}$ Marek Lubusky, ${ }^{5}$ Cruz-Martinez Rogelio, ${ }^{6}$ Cruz-Lemini Mónica, ${ }^{6}$ Martinez-Rodriguez Miguel, ${ }^{6}$ Pamela Socias, ${ }^{7}$ Cristina Aleuanlli, ${ }^{7}$ Mauro C Parra Cordero ${ }^{7}$

To cite: Figueras F, Gratacos E, Rial M, et al. Revealed versus concealed criteria for placental insufficiency in an unselected obstetric population in late pregnancy (RATI037): randomised controlled trial study protocol. BMJ Open 2017;7:e014835. doi:10.1136/ bmjopen-2016-014835

Received 24 0ctober 2016 Revised 29 January 2017 Accepted 23 February 2017

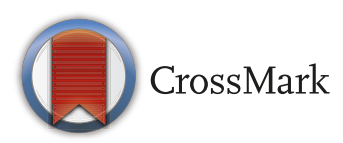

For numbered affiliations see end of article.

Correspondence to Dr Francesc Figueras; ffiguera@clinic.cat

\section{ABSTRACT}

Introduction Fetal growth restriction (FGR) affects $5 \%-10 \%$ of all pregnancies, contributing to $30 \%-50 \%$ of stillbirths. Unfortunately, growth restriction often is not detected antenatally. The last weeks of pregnancy are critical for preventing stillbirth among babies with FGR because there is a pronounced increase in stillbirths among growth-restricted fetuses after 37 weeks of pregnancy. Here we present a protocol (V.1, 23 May 2016) for the RATI037 trial, which evaluates an integrated strategy for accurately selecting at-risk fetuses for delivery at term. The protocol is based on the combination of fetal biometry and cerebroplacental ratio (CPR). The primary objective is to reduce stillbirth rates. The secondary aims are to detect low birth weights and adverse perinatal outcomes.

Methods and analysis The study is designed as multicentre (Spain, Chile, Mexico,Czech Republic and Israel), open-label, randomised trial with parallel groups. Singleton pregnancies will be invited to participate after routine second-trimester ultrasound scan $\left(19^{+0}-22^{+6}\right.$ weeks of gestation), and participants will be randomly allocated to receive revealed or concealed CPR evaluation. Then, a routine ultrasound and Doppler scan will be performed at $36^{+0}-37^{+6}$ weeks. Sociodemographic and clinical data will be collected at enrolment. Ultrasound and Doppler variables will be recorded at $36^{+0}-37^{+6}$ weeks of pregnancy. Perinatal outcomes will be recorded after delivery. Univariate (with estimated effect size and its 95\% $\mathrm{Cl}$ ) and multivariate (mixed-effects logistic regression) comparisons between groups will be performed. Ethics and dissemination The study will be conducted in accordance with the principles of Good Clinical Practice. This study was accepted by the Clinical Research Ethics Committee of Hospital Clinic Barcelona on 23May 2016. Subsequent approval by individual ethical committees and competent authorities was granted. The study results will be published in peer-reviewed journals and disseminated at international conferences.

Trial registration number NCT02907242; pre-results.
Strengths and limitations of this study

- This is the first randomised study examining the impact on perinatal heath of evaluating the fetal cerebroplacental ratio to select patients for labour induction.

- The sample size is powered to detect clinically relevant reductions in hard adverse perinatal events, including perinatal death.

- The generalisability of this study is supported by the multicentre design, covering medium-income to high-income settings.

- The nature of the intervention makes it impossible to blind participants,

Follow-up of the offspring is limited to the neonatal period.

\section{INTRODUCTION}

Background and rationale

Fetal growth restriction (FGR) is defined as a failure to reach the growth potential. FGR affects $5 \%-10 \%$ of all pregnancies and contributes to $30 \%-50 \%$ of stillbirths. ${ }^{1}$ In most cases, FGR is due to placental insufficiency. ${ }^{2}$ Population-based studies have endorsed not only the linkage between FGR and increased risk of mortality and morbidity in the perinatal period, but the risk of delayed consequences into childhood and adulthood..$^{3-5}$ Therefore, antenatal identification of FGR is crucial to optimise the timing of delivery and to reduce perinatal risks. ${ }^{56}$ Unfortunately, growth restriction often is not detected antenatal. During routine clinical practice, as many as $75 \%$ of babies at risk for FGR are not recognised before delivery. ${ }^{7}$ Notably, the gestational age at birth of FGR-related but undetected stillbirths is 280 days, 
while it is 270 days in detected cases. ${ }^{5}$ This indicates that the last weeks of pregnancy are critical to prevent stillbirth for FGR babies. This conclusion is further supported by large-scale studies showing that there is a pronounced increase in stillbirths among small fetuses after 37 weeks of pregnancy. ${ }^{8}$

\section{Antenatal screening for small for gestational age/FGR}

The first step to detect FGR due to placental insufficiency is to detect fetuses at risk for small for gestational age (SGA). SGA at birth is defined as birth weight below the 10th percentile for local standards at each study site. The typical third trimester strategy to monitor growth involves fetal weight measurement. However, most SGA infants are not detected in low-risk populations using this strategy. ${ }^{9}$ Although the use of strictly defined methodology and maternal-customised standards could potentially improve fetal weight measurement, ${ }^{10}$ evidence supporting its use is lacking. Routine third trimester ultrasound scan performs better than selective scans based on risk factors (evidence level IB) ${ }^{11}$ In addition, one random controlled trial reported that a 36-week scan provided better prediction of SGA at birth than a 32-week scan. ${ }^{12}$ The risk of delaying the scan after 36 weeks is that the stillbirth rate increases among SGA babies after 37 weeks. ${ }^{8}$

Once SGA is suspected, healthcare providers should determine whether the fetus has constitutional smallness or true FGR using one of several possible strategies. The rationale of customisation adjusts for fetal growth standards with respect to maternal characteristics that influence fetal size, such as parity, maternal weight and height and ethnicity. By determining an individualised growth potential for each fetus, constitutional smallness could be differentiated from FGR. Although this concept has been shown to be useful in neonates to predict adverse neonatal outcomes, ${ }^{13}$ customised standards have not been shown to improve the detection of placental insufficiency in fetuses. ${ }^{14}$

Fetuses destined to have an adverse outcome have a slower growth rate than those with normal outcomes. ${ }^{15}$ In SGA babies, some studies have found that growth rate performs better as a marker than other fetal and maternal parameters. ${ }^{11} 16$ However, there is controversy regarding the best methodology to use for longitudinal growth measurement, and multiple strategies have been used including conditional assessment, individualised longitudinal growth, growth rates and growth trajectories. Another limitation of longitudinal growth is that assessments with intervals $<3$ weeks are prone to result in a large proportion of false positives. ${ }^{17}$ This problem is critical when SGA is diagnosed near term because there is not enough time remaining to properly assess longitudinal growth.

In early-intrauterine growth restriction, Doppler assessment of the umbilical artery (UA) can reduce perinatal mortality and morbidity, ${ }^{18}$ and is a standard of care in fetal monitoring. By contrast, in late SGA, the UA does not accurately reflect placental insufficiency. ${ }^{19}$ Under hypoxic conditions, the fetus redistributes its flow towards the brain, a phenomenon called brain sparing. This is reflected in the middle cerebral artery (MCA) flow, which shows decreased pulsatility during Doppler evaluation. A proportion of late SGA babies develop abnormal MCA and, in a more pronounced way, abnormal cerebroplacental ratio (CPR) ${ }^{20}$ which is the ratio between MCA and UA pulsatility index (PI). The evidence suggests that SGA babies with abnormal CPR have a 10-fold increased risk of adverse outcome, ${ }^{21}$ which indicates that CPR is a useful marker to differentiate constitutional smallness from late FGR.

\section{Management and delivery of late-SGA babies}

A random controlled trial shows that systematic induction of late-SGA babies at 37-38 weeks does not improve perinatal and neurodevelopmental outcomes. ${ }^{22}{ }^{23}$ However, induction at this gestational age does not worsen neonatal outcomes and may be more cost-effective than later induction; therefore, most guidelines recommend delivery of SGA babies at risk of placental insufficiency (growth-restricted babies) after 37-38weeks, which prevents those rare but devastating cases of stillbirth that occur during the last weeks of pregnancy. There is consensus that abnormal CPR is a defining criterion for FGR in SGA babies. $^{24}$

\section{Only some FGR babies are small}

It is biologically implausible that all cases of placental insufficiency occurred in babies with birth weights below the 10th percentile. In fact, perinatal mortality remains higher in babies between the 10th and the 50th percentile of birth weight, ${ }^{25}$ suggesting that a proportion of cases of placental insufficiency exhibit growth within normal ranges. There is evidence showing that normally growing babies with abnormal CPR have a higher frequency of placental insufficiency as determined by abnormal uterine artery Doppler, ${ }^{26}$ which may impair their neurobehaviour at birth. ${ }^{27}$ Recent work shows that CPR is associated with perinatal mortality, independently of birth weight. ${ }^{28}$ Finally, there is evidence that abnormal CPR before birth is associated with the occurrence of fetal distress during labour, ${ }^{29}$ supporting the concept that CPR reflects latent placental insufficiency.

\section{Justification for the study}

Although fetal size and CPR independently correlate with adverse perinatal outcome, it has not been determined whether a strategy of labour induction based on these parameters has the potential to prevent stillbirths. It is necessary to obtain evidence in support of this hypothesis before recommending the widespread use of this strategy.

\section{HYPOTHESIS}

A proportion of fetuses with normal growth (as defined per current standards) has placental insufficiency and restricted growth potential. These fetuses exhibit biophysical changes that can be detected by abnormal CPR. The 
combination of abnormal CPR with fetal biometry to detect fetuses affected by FGR could identify the group of at-risk babies. Labour induction at term may prevent adverse outcomes for these babies.

\section{OBJECTIVES}

\section{Primary objective}

The primary objective is to determine whether the addition of CPR Doppler evaluation to the standard ultrasound biometrical measurements can reduce perinatal mortality by efficiently selecting women for labour induction.

\section{Secondary objectives}

i. to determine whether the addition of CPR Doppler evaluation to the standard ultrasound biometrical measurements decreases mild and severe adverse perinatal outcomes after labour induction;

ii. to determine whether the addition of CPR Doppler evaluation to the standard ultrasound biometrical measurements decreases neurological morbidity after labour induction;

iii. to determine whether the addition of CPR Doppler evaluation to the standard ultrasound biometrical measurements decreases non-neurological morbidity (defined as prolonged neonatal intensive care unit (NICU) stay without criteria of neurological morbidity) after labour induction;

iv. to evaluate the predictive capacity of an integrated strategy based on the combination of fetal biometries and CPR at 36-37weeks to detect prenatal low birth weight.

\section{METHODS}

\section{Study setting}

The study will be conducted within academic hospitals with specialist experience in managing FGR, in Spain (Hospital Clinic Barcelona), Chile (Hospital Clinico
Universitario de Santiago Chile), Mexico (Hospital of Querétaro) Czech Republic (Palacky University Hospital in Olomouc, Charles University Hospital in Prague) and Israel (Lis Hospital for Women, Tel Aviv). Each of these hospitals attends $>3000$ deliveries and performs $>10000$ fetal ultrasounds per year.

\section{Trial design}

This is a multicentre, open-label, randomised trial with parallel groups. The study design adheres to the SPIRIT quality standard criteria for randomised trials. ${ }^{30}$

\section{Eligibility criteria}

The following five criteria were used for inclusion in the study: (i) viable singleton non-malformed fetus at the routine second trimester ultrasound, (ii) available first trimester scan (with embryo crown-rump length measurement), (iii) absence of adverse medical or obstetric history, (iv) maternal age at recruitment $>18$ years and (v) capacity to give informed consent. The following three criteria were used for exclusion from the study: (i) abnormal karyotype, (ii) structural abnormalities and (iii) congenital infections.

\section{Exclusion criteria}

Women with obstetrical complication arising before 37 weeks (as premature rupture of membranes (PROM)) prompting delivery will be excluded.

\section{Intervention}

Trial participants will be allocated to revealed or concealed third trimester (36-37weeks) CPR evaluation. In the concealed group, management will adhere to the current standard for managing SGA pregnancies. In the revealed group, CPR data obtained from Doppler evaluation will be integrated into the criteria used for decisions regarding labour induction. The flow chart in figure 1 illustrates the management strategy following intervention.

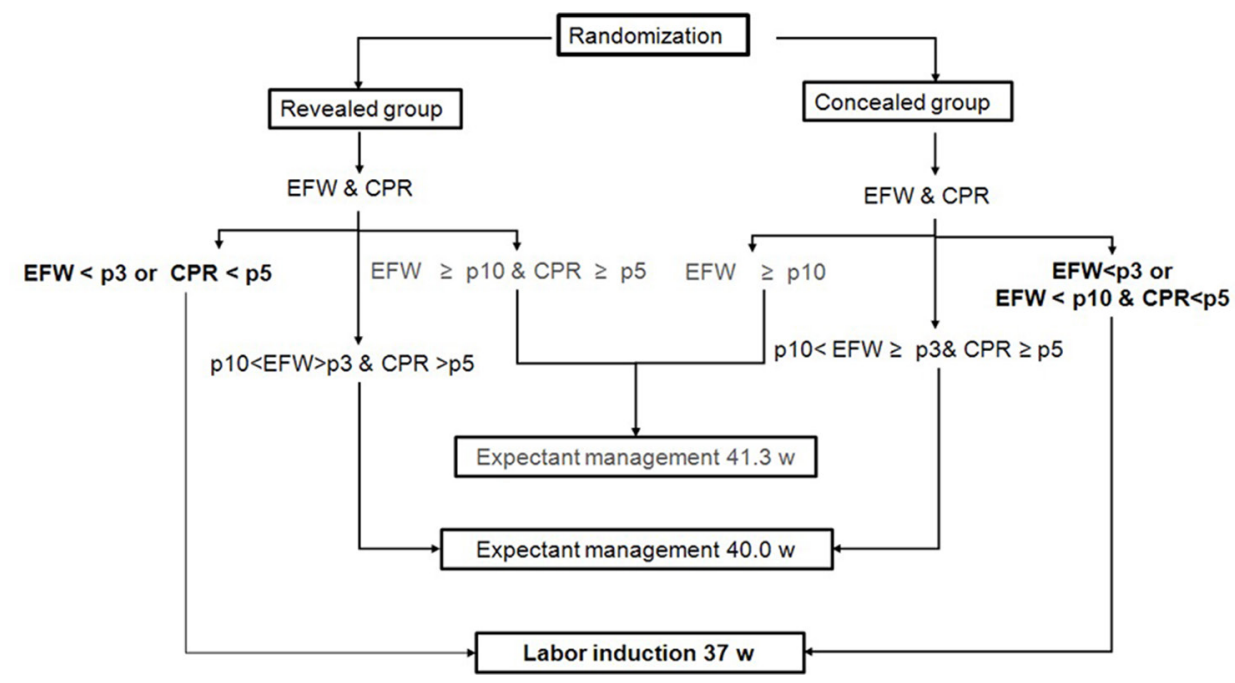

Figure 1 Flow chart of study intervention and management. CPR, cerebroplacental ratio; EFW, estimated fetal weight. 


\section{Predictive variables}

The predictive variables include fetal biometries, amniotic fluid, Doppler evaluation, UAPI and MCA PI. The fetal biometries include biparietal diameter, head circumference, abdominal circumference and femur length. These will be measured according to standardised procedures. ${ }^{31}$ The Hadlock formula will be used to calculate estimated fetal weight, which will qualify for fetal SGA if it is below the 10th percentile. ${ }^{32}$ Amniotic fluid will be measured in the deepest vertical pocket (an abnormal value is defined as $<20 \mathrm{~mm}$ ). Doppler measures will be obtained in the absence of fetal movements and with voluntarily suspended maternal breathing. Doppler parameters will be measured automatically from three or more consecutively similar waveforms, with the angle of insonation as close to $0^{\circ}$ as possible. UA PI will be measured from a free-floating cord loop. MCA PI will be measured in a transversal view of the fetal head, at the level of its origin from the circle of Willis. The latter two parameters will be used to derive the CPR as follows: MCA PI/UA PI. CPR will be considered as abnormal when it is below the fifth percentile. $^{33}$

\section{OUTCOMES}

\section{Primary outcome}

The primary outcome will be perinatal mortality from inclusion to +28 neonatal days.

\section{Secondary outcomes}

Four secondary outcomes will be recorded, including mild adverse perinatal outcome, severe neurological morbidity, severe non-neurological morbidity and SGA at birth. Mild adverse perinatal outcome is defined as any of the following: (i) caesarean section for fetal distress (defined according local protocols), (ii) 5 min Apgar score $<7$, (iii) neonatal acidosis at birth (UA $\mathrm{pH}<7.15$ and base excess $>$ $-12 \mathrm{mEq} / \mathrm{L}$ ) or (iv) admission to the neonatal unit. Severe neurological morbidity is defined as any of the following: (i) seizure, ${ }^{34}$ (ii) interventricular haemorrhage $>$ grade $\mathrm{II},{ }^{35}$ (iii) periventricular leukomalacia ${ }^{36}$ or (iv) hypoxia-ischaemia or encephalopathy. ${ }^{34}$ Severe non-neurological morbidity is defined as any of the following: (i) prolonged NICU stay ( $>10$ days) without criteria of neurological morbidity, (ii) necrotising enterocolitis, ${ }^{34}$ (iii) acute renal failure (serum creatinine $>1.5 \mathrm{mg} / \mathrm{dL}$ ) or (iv) cardiac failure (requiring ionotropic agents). SGA at birth is defined as birth weight below the 10th percentile for local standards at each study site.

\section{Participant timeline}

Table 1 shows the study timeline.

\section{Sample size}

The required sample size was estimated as follows. For a $3 \%$ reduction of the stillbirth rate (from $5 \%$ o to $2 \%$ ), assuming a type I error of $5 \%$ and aiming for a power of $80 \%$, a total of 11582 participants (5791 per arm) are required. The participating centres attend a total of 12000 deliveries per year. Assuming that $50 \%$ of women are eligible and willing to participate, it is expected that the required sample size can be achieved within 2 years.

\section{Allocation}

An online service (http://www.randomization.com) was used to generate randomised sequences in blocks of 100 participants. This ensured a balanced distribution within the two study arms, stratified for participating site. The

Table 1 Study timeline

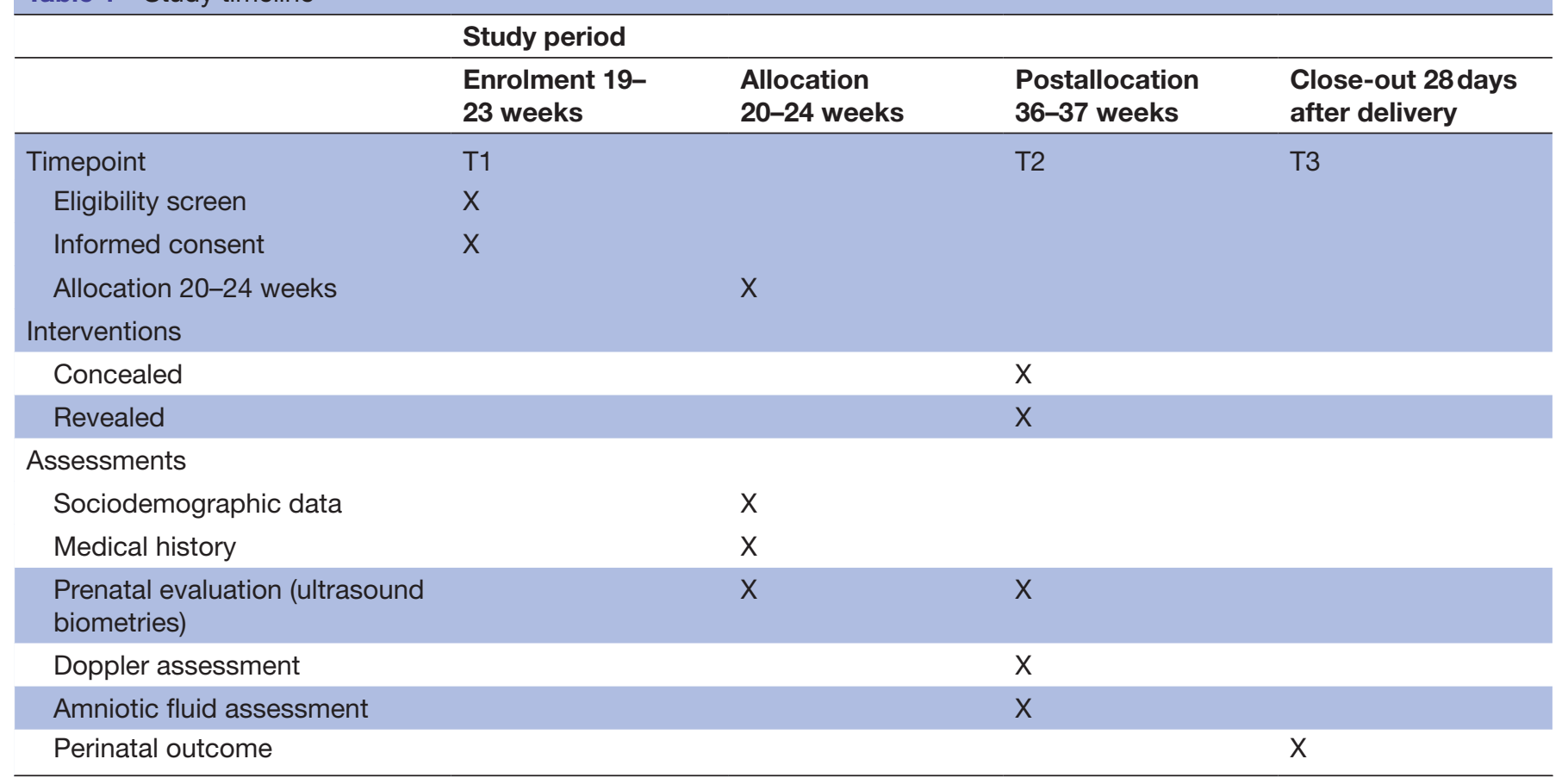


allocation sequence will be sequestered internally by a Clinical Trials Unit.

At the time of routine second trimester scans and after enrolment, recruiting physicians will obtain the allocation group from a web-based system. Women who decline consent for randomisation but authorise the use of their medical data will be included in the database. These data will be used for external validity testing of the trial. Due to the nature of the intervention, it is not possible to blind participants, obstetricians or outcome assessors with respect to the study group.

\section{Data collection}

The coordinator at each site will collect sociodemographic data and first and second trimester ultrasound data. Participants will be scheduled for a third trimester ultrasound at $36+0$ to $37+6$ weeks; these data will be used to obtain fetal biometric data, measure the amniotic fluid volume in the deepest vertical pocket and calculate CPR. Perinatal outcomes will be recorded after delivery and at +28 days.

Participant data for this study will be anonymised and entered into an electronic case report form (e-CRF) hosted in a secured website by each site coordinator. Logic and range rules operate in the e-CRF to minimise errors in entering the information.

\section{Data monitoring}

An independent Clinical Trial Unit will perform offline data auditing every 6 months to check for missing information and errors. Umbilical and middle cerebral Doppler images will be requested from a random sample of 25 women every 6 months to check for accuracy according the following criteria: (i) location site (UA free-loop and proximal portion of the MCA); (ii) size of the Doppler spectral gate (equivalent to the vessel diameter); (iii) scale velocity of the pulsed Doppler, which should result in a waveform height equivalent to at least $2 / 3$ of the $y$-axis; and (iv) automatic calculation of the PI from at least three consecutively similar waveforms. The sites will be notified of any deviation from the standard recommendations for amendment.

\section{Statistical analysis}

Statistical analysis will be based on the originally assigned groups (intention-to-treat). A binomial distribution model will be used to determine the $95 \%$ CI of proportions. Student's t-test (or non-parametric Mann-Whitney U test) and Pearson's $\chi^{2}$ test or linear-by-linear $\chi^{2}$ test (for trends across ordered categories) will be performed for univariate between-group comparisons of quantitative or qualitative variables, respectively. A mixed-effects logistic regression analysis of the incidence of stillbirth with fixed effects for allocation group and random effects for the study centre will be used. Logistic regression also will be used to determine which of the variables have a significant contribution for predicting stillbirth, low birth weight and adverse perinatal outcome. In these regression models, stepwise algorithms will be performed to select variables at p-value cut-off of 0.05 for inclusion and 0.1 for exclusion, with tolerance level $>0.01$. Goodness-of-fit models will be assessed by calculating Nagelkerke's $R^{2}$ and by performing a Hosmer and Lemeshow's test. Performance of the models also will be evaluated by constructing receiver operating characteristic (ROC) curves. A subanalysis will be carried out in patients undergoing an elective caesarean section. ROC curves will be compared pairwise using the DeLong procedure. Open-source software (The R Foundation for Statistical Computing) will be used for all computations and graph construction (R V2.15.1).

\section{Author affiliations}

${ }^{1}$ Fetal i+D Fetal Medicine Research Center, BCNatal - Barcelona Center for Maternal-Fetal and Neonatal Medicine (Hospital Clínic and Hospital Sant Joan de Deu), IDIBAPS, University of Barcelona, Barcelona, Spain

${ }^{2}$ Centre for Biomedical Research on Rare Diseases (CIBER-ER), Barcelona, Spain

${ }^{3}$ Lis Hospital for Women, Tel Aviv University, Tel Aviv, Israel

${ }^{4}$ Institute for the care of mother and child, Third Faculty of Medicine, Charles University, Prague, Czech Republic

${ }^{5}$ Departmente of Obstetrics and Gynecology, Palacky University Olomouc, Faculty of Medicine and Dentistry, University Hospital Olomouc, Olomouc, Czech Republic

${ }^{6}$ Unidad de Investigación en Medicina y Cirugía Fetal, Hospital de Especialidades del Niño y la Mujer de Querétaro. Unidad de Investigación en Neurodesarrollo, Instituto de Neurobiología, Universidad Nacional Autónoma de México (UNAM) campus Juriquilla, Querétaro, México

${ }^{7}$ Department of Obstetrics and Gynecology, Fetal Medicine Unit, University of Chile Hospital, Santiago, Chile

Contributors MR is the project monitor, contributed to the study planning and will participate in the report of the data. RC, MP, ML and LK are coordinators at their respective sites, contributed to the study planning and will participate in the report of the data. MC and MM contributed to the conducting and will report the data. FF is the general coordinator and PI of the project. EG is co-principal investigator of the study.

Funding The study was funded by the Erasmus ${ }^{+}$Programme of the European Union (Framework Agreement number: 2013-0040).

Competing interests None declared.

Ethics approval Hospital Clinic Ethics Committee.

Provenance and peer review Not commissioned; externally peer reviewed.

Open Access This is an Open Access article distributed in accordance with the Creative Commons Attribution Non Commercial (CC BY-NC 4.0) license, which permits others to distribute, remix, adapt, build upon this work non-commercially, and license their derivative works on different terms, provided the original work is properly cited and the use is non-commercial. See: http://creativecommons.org/ licenses/by-nc/4.0/

(c) Article author(s) (or their employer(s) unless otherwise stated in the text of the article) 2017. All rights reserved. No commercial use is permitted unless otherwise expressly granted.

\section{REFERENCES}

1. M Kady S, Gardosi J. Perinatal mortality and fetal growth restriction. Best Pract Res Clin Obstet Gynaecol 2004;18:397-410.

2. Lackman F, Capewell V, Gagnon R, et al. Fetal umbilical cord oxygen values and birth to placental weight ratio in relation to size at birth. Am J Obstet Gynecol 2001;185:674-82.

3. Crispi F, Bijnens B, Figueras F, et al. Fetal growth restriction results in remodeled and less efficient hearts in children. Circulation 2010;121:2427-36.

4. Mclntire DD, Bloom SL, Casey BM, et al. Birth weight in relation to morbidity and mortality among newborn infants. N Engl J Med 1999;340:1234-8.

5. Gardosi J, Madurasinghe V, Williams M, et al. Maternal and fetal risk factors for stillbirth: population based study. BMJ 2013;346:f108. 
6. Lindqvist PG, Molin J. Does antenatal identification of smallfor-gestational age fetuses significantly improve their outcome? Ultrasound Obstet Gynecol 2005;25:258-64.

7. Hepburn M, Rosenberg K. An audit of the detection and management of small-for-gestational age babies. Br J Obstet Gynaecol 1986;93:212-6.

8. Pilliod RA, Cheng YW, Snowden JM, et al. The risk of intrauterine fetal death in the small-for-gestational-age fetus. Am J Obstet Gynecol 2012;207:318.e1-318.e6.

9. Kayem G, Grangé G, Bréart G, et al. Comparison of fundal height measurement and sonographically measured fetal abdominal circumference in the prediction of high and low birth weight at term. Ultrasound Obstet Gynecol 2009;34:566-71.

10. Gardosi J, Giddings S, Clifford S, et al. Association between reduced stillbirth rates in England and regional uptake of accreditation training in customised fetal growth assessment. BMJ Open 2013;3:e003942.

11. Sovio U, White IR, Dacey A, et al. Screening for fetal growth restriction with universal third trimester ultrasonography in nulliparous women in the Pregnancy Outcome Prediction (POP) study: a prospective cohort study. Lancet 2015;386:2089-97.

12. Roma E, Arnau A, Berdala R, et al. Ultrasound screening for fetal growth restriction at 36 vs 32 weeks' gestation: a randomized trial (ROUTE). Ultrasound Obstet Gynecol 2015;46:391-7.

13. Clausson B, Gardosi J, Francis A, et al. Perinatal outcome in SGA births defined by customised versus population-based birthweight standards. BJOG 2001:108:830-4.

14. Larkin JC, Hill LM, Speer PD, et al. Risk of morbid perinatal outcomes in small-for-gestational-age pregnancies: customized compared with conventional standards of fetal growth. Obstet Gynecol 2012;119:21-7.

15. de Jong CL, Francis A, van Geijn HP, et al. Fetal growth rate and adverse perinatal events. Ultrasound Obstet Gynecol 1999;13:86-9.

16. Chang TC, Robson SC, Spencer JA, et al. Prediction of perinatal morbidity at term in small fetuses: comparison of fetal growth and Doppler ultrasound. Br J Obstet Gynaecol 1994;101:422-7.

17. Mongelli M, Ek S, Tambyrajia R. Screening for fetal growth restriction: a mathematical model of the effect of time interval and ultrasound error. Obstet Gynecol 1998;92:908-12.

18. Neilson JP, Alfirevic Z. Doppler ultrasound for fetal assessment in high risk pregnancies. Cochrane Database Syst Rev 2000;2:CD000073.

19. Parra-Saavedra M, Simeone S, Triunfo S, et al. Correlation between placental underperfusion, histologic signs and perinatal morbidity in late-onset small for gestational age fetuses. Ultrasound Obstet Gynecol 2013;45.

20. Oros D, Figueras F, Cruz-Martinez R, et al. Longitudinal changes in uterine, umbilical and fetal cerebral Doppler indices in late-onset small-for-gestational age fetuses. Ultrasound Obstet Gynecol 2011;37:191-5.
21. Nassr AA, Abdelmagied AM, Shazly SA. Fetal cerebro-placental ratio and adverse perinatal outcome: systematic review and meta-analysis of the association and diagnostic performance. J Perinat Med 2016;44:249-56.

22. van Wyk L, Boers KE, van der Post JA, et al. Effects on (neuro) developmental and behavioral outcome at 2 years of age of induced labor compared with expectant management in intrauterine growthrestricted infants: long-term outcomes of the DIGITAT trial. Am J Obstet Gynecol 2012;206:406.e1-406.e7.

23. Boers KE, Vijgen SM, Bijlenga D, et al. Induction versus expectant monitoring for intrauterine growth restriction at term: randomised equivalence trial (DIGITAT). BMJ 2010;341:c7087.

24. Gordijn SJ, Beune IM, Thilaganathan B, et al. Consensus definition of fetal growth restriction: a Delphi procedure. Ultrasound Obstet Gynecol 2016;48:333-9.

25. Vasak B, Koenen SV, Koster MP, et al. Human fetal growth is constrained below optimal for perinatal survival. Ultrasound Obstet Gynecol 2015;45:162-7.

26. Khalil A, Morales-Rosello J, Townsend R, et al. Are fetal cerebroplacental ratio and impaired placental perfusion recorded in the third trimester predictors of stillbirth and perinatal loss? Ultrasound Obstet Gynecol 2015.

27. Mula R, Savchev S, Parra M, et al. Increased fetal brain perfusion and neonatal neurobehavioral performance in normally grown fetuses. Fetal Diagn Ther 2013;33:182-8.

28. Khalil AA, Khan N, Bowe S, et al. Discordance in fetal biometry and Doppler are independent predictors of the risk of perinatal loss in twin pregnancies. Am J Obstet Gynecol 2015;213:222.e1-222.e10.

29. Prior $T$, Mullins $E$, Bennett $P$, et al. Prediction of intrapartum fetal compromise using the cerebroumbilical ratio: a prospective observational study. Am J Obstet Gynecol 2013;208:124.e1-124. e6.

30. Antes G. The new CONSORT statement. BMJ 2010;340:c1432.

31. Salomon LJ, Alfirevic Z, Berghella V, et al. Practice guidelines for performance of the routine mid-trimester fetal ultrasound scan. Ultrasound Obstet Gynecol 2011;37:116-26.

32. Figueras F, Meler E, Iraola A, et al. Customized birthweight standards for a Spanish population. Eur J Obstet Gynecol Reprod Biol 2008;136:20-4.

33. Baschat AA, Gembruch U. The cerebroplacental Doppler ratio revisited. Ultrasound Obstet Gynecol 2003;21:124-7.

34. Vermont Oxford Network Database. Manual of Operations For Infants Born in 2006. 2005. Release 10.1.

35. Papile LA, Burstein J, Burstein R, et al. Incidence and evolution of subependymal and intraventricular hemorrhage: a study of infants with birth weights less than 1,500 gm. J Pediatr 1978;92:529-34.

36. De Vries LS, Regev R, Pennock JM, et al. Ultrasound evolution and later outcome of infants with periventricular densities. Early Hum Dev 1988;16(2-3):225-33. 
Correction: Revealed versus concealed criteria for placental insufficiency in an unselected obstetric population in late pregnancy (RATIO37): randomised controlled trial study protocol

Figueras F, Gratacos E, Rial M, et al. Revealed versus concealed criteria for placental insufficiency in an unselected obstetric population in late pregnancy (RATIO37): randomised controlled trial study protocol. BMJ Open 2017;7:e14835. doi: 10.1136/ bmjopen-2016-014835.

This article was previously published with an error in the author list.

Two co-authors were missed off the original author list, Eran Ashwal and Kacerovsky, Marian.

Eran Ashwal's affiliation is Lis Hospital for Women, Tel Aviv University, Tel Aviv, Israel. Marian Kacerovsky's affiliation is Department of Obstetrics and Gynecology, Charles University in Prague, Faculty of Medicine Hradec Kralove, University Hospital Hradec Kralove, Hradec Kralove, Czech Republic.

The author order is also incorrect.

The updated author list should be as follows:

Figueras, Francesc

Gratacos, Eduard

Rial, Marta

Gull, Ilan

Eran, Ashwal

Kacerovsky, Marian

Lubusky, Marek

Krofta, Ladislav

Cruz, Rogelio

Cruz, Monica

Martinez, Miguel

Parra, Mauro

Socias, Pamela

Aleuanlli, Maria Cristina

Open access This is an open access article distributed in accordance with the Creative Commons Attribution Non Commercial (CC BY-NC 4.0) license, which permits others to distribute, remix, adapt, build upon this work non-commercially, and license their derivative works on different terms, provided the original work is properly cited, appropriate credit is given, any changes made indicated, and the use is non-commercial. See: http://creativecommons.org/licenses/by-nc/4.0/.

(c) Author(s) (or their employer(s)) 2018. Re-use permitted under CC BY-NC. No commercial re-use. See rights and permissions. Published by BMJ.

BMJ Open 2018;9:e014835corr1. doi:10.1136/bmjopen-2016-014835corr1

Check for updates 